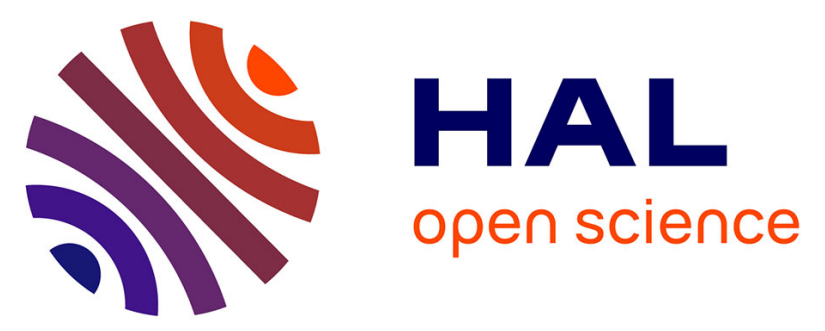

\title{
An exact local error representation of exponential operator splitting methods for evolutionary problems and applications to linear Schrödinger equations in the semi-classical regime.
}

\author{
Stéphane Descombes, Mechthild Thalhammer
}

\section{To cite this version:}

Stéphane Descombes, Mechthild Thalhammer. An exact local error representation of exponential operator splitting methods for evolutionary problems and applications to linear Schrödinger equations in the semi-classical regime.. BIT Numerical Mathematics, 2010, 50 (4), pp.729-749. 10.1007/s10543010-0282-4 . hal-00550259

\section{HAL Id: hal-00550259 \\ https://hal.science/hal-00550259}

Submitted on 25 Dec 2010

HAL is a multi-disciplinary open access archive for the deposit and dissemination of scientific research documents, whether they are published or not. The documents may come from teaching and research institutions in France or abroad, or from public or private research centers.
L'archive ouverte pluridisciplinaire HAL, est destinée au dépôt et à la diffusion de documents scientifiques de niveau recherche, publiés ou non, émanant des établissements d'enseignement et de recherche français ou étrangers, des laboratoires publics ou privés. 


\title{
An exact local error representation of exponential operator splitting methods for evolutionary problems and applications to linear Schrödinger equations in the semi-classical regime
}

\author{
Stéphane Descombes and Mechthild Thalhammer
}

\begin{abstract}
In this paper, we are concerned with the derivation of a local error representation for exponential operator splitting methods when applied to evolutionary problems that involve critical parameters. Employing an abstract formulation of differential equations on function spaces, our framework includes Schrödinger equations in the semi-classical regime as well as parabolic initial-boundary value problems with high spatial gradients. We illustrate the general mechanism on the basis of the first-order Lie splitting and the second-order Strang splitting method. Further, we specify the local error representation for a fourth-order splitting scheme by Yoshida. From the given error estimate it is concluded that higher-order exponential operator splitting methods are favourable for the time-integration of linear Schrödinger equations in the semi-classical regime with critical parameter $0<\varepsilon<<1$, provided that the time stepsize $h$ is sufficiently smaller than $\sqrt[p]{\varepsilon}$, where $p$ denotes the order of the splitting method.
\end{abstract}

Keywords Evolutionary equations - Time-dependent Schrödinger equations · Exponential operator splitting methods · Local error representation

Mathematics Subject Classification (2000) 65L05 - 65M12 · 65J10

\section{Introduction}

In this paper, we are concerned with exponential operator splitting methods $[2,5-7$, $9,14,18,19,21,24,26]$ for the time-integration of abstract evolutionary problems [11, $16,17,20,23]$. Our objective is the specification and inspection of an alternative local error representation; such a representation provides the basis for a convergence analysis of splitting methods when applied to (stiff) differential equations. Compared to

Stéphane Descombes, Laboratoire J. A. Dieudonné, Université de Nice - Sophia Antipolis, Parc Valrose, 06108 Nice Cedex 02, France. Mechthild Thalhammer, Institut für Mathematik, Leopold-Franzens Universität Innsbruck, 6020 Innsbruck, Austria. E-mail: sdescomb@unice.fr, mechthild.thalhammer@uibk.ac.at 
other representations of the local error that rely on the well-known Baker-CampbellHausdorff formula or on techniques exploited in $[13,18,19,22,26]$, respectively, the presently considered representation along the lines of $[9,10]$ is particularly suitable for the investigation of evolutionary problems that involve critical parameters.

The incentive for the present work originates from the question whether exponential operator splitting methods of higher order are favourable for evolutionary Schrödinger equations in the semi-classical regime; our interest in this theme is motivated by theoretical and numerical investigations for the first-order Lie-Trotter splitting and the second-order Strang splitting provided by $[3,4,12]$, see also the references given therein. In the present paper, we follow an approach which yields optimal global error bounds with respect to critical parameters. Further, it facilitates a specification of the involved quantities and therefore might allow to prescribe values of the time stepsizes for given tolerances. It is notable that our abstract framework includes evolutionary Schrödinger equations in the semi-classical regime as well as parabolic initial-boundary value problems involving high spatial gradients.

As a model problem, we consider the following linear Schrödinger equation for a function $\psi: \mathbb{R}^{d} \times \mathbb{R}_{\geq 0} \rightarrow \mathbb{C}$

$$
\begin{gathered}
\text { i } \varepsilon \partial_{t} \psi(x, t)=-\varepsilon^{2} \Delta \psi(x, t)+U(x) \psi(x, t), \\
\psi(x, 0) \text { given, } \quad x \in \mathbb{R}^{d}, \quad t \geq 0 .
\end{gathered}
$$

Employing an abstract formulation, problem (1.1) takes the form

$$
u^{\prime}(t)=A u(t)+B u(t), \quad t \geq 0, \quad u(0) \text { given, }
$$

with unbounded linear operator $A$ comprising the Laplacian, i.e., $A=\mathrm{i} \varepsilon \Delta$, and multiplication operator $B$ defined by the potential, i.e. $B=-\mathrm{i} U / \varepsilon$. Any exponential operator splitting method for (1.2) can be cast into the form

$$
u_{n+1}=\prod_{j=1}^{s} \mathrm{e}^{h_{n} b_{j} B} \mathrm{e}^{h_{n} a_{j} A} u_{n}, \quad n \geq 0, \quad u_{0} \text { given, }
$$

yielding numerical approximations $u_{n+1} \approx u\left(t_{n+1}\right)=u\left(t_{n}+h_{n}\right)$ at certain time grid points.

For nonlinear Schrödinger equations such as the Gross-Pitaevskii equation describing the order parameter of a Bose-Einstein condensate (with $\varepsilon=1$ in (1.1) and additional nonlinearity $\vartheta|\psi(x, t)|^{2} \psi(x, t)$ for some $\vartheta \in \mathbb{R}$ ), numerical comparisons given in $[2,5,7,24]$, e.g., show that higher-order splitting schemes are superior to standard integrators when low tolerances are required or a long-term integration is carried out. These numerical observations are also confirmed by theoretical investigations.

For instance, for an exponential operator splitting method of (classical) order $p$, applied to a linear evolutionary Schrödinger equation involving a sufficiently regular bounded potential, the local error expansion exploited in $[18,26]$ leads to a global error estimate of the form

$$
\left\|u_{N}-u\left(t_{N}\right)\right\|_{L^{2}} \leq C\left(\left\|u_{0}-u(0)\right\|_{L^{2}}+\sum_{n=0}^{N-1} h_{n}^{p+1}\|u(0)\|_{H^{p}}\right) .
$$


It is also considered possible to extend the techniques used in $[13,18,19,22,26]$ to establish estimates for splitting methods of arbitrarily high order applied to nonlinear problems.

Unfortunately, for small parameter values $0<\varepsilon<<1$, the above mentioned approach is not appropriate to provide optimal bounds with respect to $\varepsilon$. For instance, for the second-order Strang splitting, the local error $u_{1}-u\left(h_{0}\right)$ (assuming $u_{0}=u(0)$ ) comprises terms such as

$$
\int_{0}^{h_{0}} \int_{0}^{\tau_{1}} \int_{0}^{\tau_{2}} \mathrm{e}^{\left(h_{0}-\tau_{1}\right) A} B \mathrm{e}^{\left(\tau_{1}-\tau_{2}\right) A} B \mathrm{e}^{\left(\tau_{2}-\tau_{3}\right) A} B u\left(t_{n-1}+\tau_{3}\right) \mathrm{d} \tau_{3} \mathrm{~d} \tau_{2} \mathrm{~d} \tau_{1}=\mathscr{O}\left(\frac{h_{0}^{3}}{\varepsilon^{3}}\right) .
$$

Contrary to this relation, numerical simulations given in [3] indicate a less restrictive dependence of the local error on the critical parameter $0<\varepsilon<<1$, namely $u_{1}$ $u\left(h_{0}\right)=\mathscr{O}\left(h_{0}^{3} / \varepsilon\right)$. Thus, the techniques exploited in the present work are needed for a better theoretical understanding of the convergence behaviour of higher-order splitting methods and the dependence of the admissible temporal stepsize on $\varepsilon$.

The structure of this work is as follows. In Section 2, we state the abstract evolutionary problem and specify the considered exponential operator splitting methods. Section 3 is devoted to the derivation of an appropriate local error representation involving multiple integrals of functions that comprise iterated commutators. From this representation and further Taylor series expansions of the integrands, as a secondary result, the (classical) order conditions are retained in Section 4. In our presentation, to illustrate the general mechanism, we focus on three example methods, namely, the first-order Lie-Trotter splitting, the second-order symmetric Lie-Trotter splitting, often refered to as Strang splitting, and a fourth-order splitting scheme involving four compositions which was proposed by Yoshida, see also [14, 21, 25,27]. Theoretical and numerical illustrations of linear Schrödinger equations in the semiclassical regime, under periodic boundary conditions and involving a sufficiently regular bounded potential, are finally given in Section 5 .

The investigation of our local error representation for nonlinear problems within a particular scope of applications is the subject of future studies.

\section{Splitting methods for evolutionary problems}

In view of a local error analysis of high-order exponential operator splitting methods for Schrödinger equations such as (1.1), it is convenient to employ an abstract formulation of the partial differential equation as an ordinary differential equation on a function space; we refer to $[13,18,22,26]$ and the references given therein for details on evolutionary Schrödinger equations.

We remark that our abstract setting also permits to incorporate initial-boundary value problems of parabolic type by utilising the framework of sectorial operators and analytic semigroups [16,20,23], see also [9]. However, carefulness is demanded with high-order schemes comprising negative method coefficients. Instead, higher-order methods involving complex coefficients can be used, see the recent works [8,15]; we note that splitting methods with complex times are also included in our approach. 
In the following, we tacitly require the arising unbounded operators and compositions thereof to be defined on suitably chosen subsets of the underlying Banach space so that all expressions remain well-defined on these domains.

\subsection{Evolutionary problems}

Henceforth, we consider linear initial value problems of the form (1.2) with (unbounded) linear operators $A: D(A) \subset X \rightarrow X$ and $B: D(B) \subset X \rightarrow X$ defined on suitable chosen subspaces of the underlying Banach space $X$. We tacitly suppose $D(A) \cap D(B)$ to be a non-empty set. The exact solution of the evolutionary problem (1.2) is (formally) given by

$$
u(t)=\mathscr{E}(t) u(0), \quad \mathscr{E}(t)=\mathrm{e}^{t(A+B)}, \quad t \geq 0 .
$$

In regard to the time-dependent Schrödinger equation (1.1), we are primarily interested in the case where $A: D(A) \subset L^{2}(\Omega) \rightarrow L^{2}(\Omega)$ is related to the $d$-dimensional Laplacian and $B$ acts as a (bounded) multiplication operator on $L^{2}(\Omega)$ for a domain $\Omega \subset \mathbb{R}^{d}$, see also $[18,22,26]$.

\subsection{High-order splitting methods}

For the time-integration of (1.2), we apply an exponential operator splitting method of (classical) order $p \geq 1$; henceforth, we employ the following general form that includes various methods proposed in literature, see also [14,21].

For a sequence of time grid points $0=t_{0}<t_{1}<\cdots<t_{N}$, the associated time stepsizes are given by $h_{n}=t_{n+1}-t_{n}$ for $0 \leq n \leq N-1$. Starting from an initial value $u_{0} \approx u(0)$, numerical approximations $u_{n+1}$ to the exact solution values $u\left(t_{n+1}\right)$ are determined through the recurrence relation

$$
u_{n+1}=\mathrm{e}^{h_{n} b_{s} B} \mathrm{e}^{h_{n} a_{s} A} \cdots \mathrm{e}^{h_{n} b_{2} B} \mathrm{e}^{h_{n} a_{2} A} \mathrm{e}^{h_{n} b_{1} B} \mathrm{e}^{h_{n} a_{1} A} u_{n}, \quad 0 \leq n \leq N-1,
$$

involving (real or complex) method coefficients $\left(a_{j}, b_{j}\right)_{j=1}^{s}$; we tacitly assume the above procedure to be well-defined on a certain domain. Employing the abbreviations

$$
\mathscr{S}_{j}(t)=\mathrm{e}^{b_{j} t B} \mathrm{e}^{a_{j} t A}, \quad \mathscr{S}_{i}^{k}=\prod_{j=i}^{k} \mathscr{S}_{j}, \quad \mathscr{S}=\mathscr{S}_{1}^{s},
$$

the exponential operator splitting method rewrites as

$$
u_{n+1}=\mathscr{S}\left(h_{n}\right) u_{n}, \quad 0 \leq n \leq N-1
$$

here, the product is defined downwards, see also (2.7).

Details on the numerical realisation of splitting methods for Schrödinger equations by using pseudo-spectral space discretisations are given in $[7,13,18]$, see also the references given therein. 
Low-order example methods that can be cast in the form (2.2) are the first-order Lie-Trotter splitting, where $p=1$ and

$$
\begin{gathered}
s=1, \quad a_{1}=1=b_{1}, \quad \text { or } \\
s=2, \quad a_{1}=b_{2}=0, \quad a_{2}=b_{1}=1,
\end{gathered}
$$

respectively, and the widely used second-order Strang splitting, where $p=2$ and

$$
\begin{gathered}
s=2, \quad a_{1}=a_{2}=\frac{1}{2}, \quad b_{1}=1, \quad b_{2}=0, \quad \text { or } \\
s=2, \quad a_{1}=0, \quad a_{2}=1, \quad b_{1}=b_{2}=\frac{1}{2},
\end{gathered}
$$

respectively. Throughout, we focus on the Lie-Trotter splitting method (2.3a); the corresponding results for (2.3b) are obtained by exchanging the roles of $A$ and $B$.

Higher-order example methods proposed in literature are reviewed in [14,21], see also $[8,15]$. As an illustration, we consider a fourth-order method by Yoshida [14, p. 40, Formula (4.4)] with real coefficients

$$
\begin{gathered}
a_{1}=0, \quad a_{2}=a_{4}=\gamma_{1}=\frac{1}{2-\sqrt[3]{2}}, \quad a_{3}=\gamma_{2}=-\frac{\sqrt[3]{2}}{2-\sqrt[3]{2}}, \\
b_{1}=b_{4}=\frac{1}{2} \gamma_{1}, \quad b_{2}=b_{3}=\frac{1}{2}\left(\gamma_{1}+\gamma_{2}\right) .
\end{gathered}
$$

Compared to other fourth-order schemes with improved accuracy and efficiency, see [6], e.g., the above scheme comprises four compositions only; thus, for (2.5) it is less involved to carry out the local error expansion of Theorem 1 by hand, obtaining expressions of a reasonable length, see also Section 4.

\subsection{Auxiliary results}

For the subsequent sections, it it convenient to employ the following auxiliary notations and results.

Let $I$ denote the identity operator and $\left(L_{j}\right)_{j}$ a family of (linear) operators. Important tools in our local error representation are iterated commutators, defined by recurrence through

$$
\operatorname{ad}_{L_{1}}^{j+1}\left(L_{2}\right)=\left[L_{1}, \operatorname{ad}_{L_{1}}^{j}\left(L_{2}\right)\right]=L_{1} \operatorname{ad}_{L_{1}}^{j}\left(L_{2}\right)-\operatorname{ad}_{L_{1}}^{j}\left(L_{2}\right) L_{1}, \quad j \geq 0,
$$

where $\operatorname{ad}_{L_{1}}^{0}\left(L_{2}\right)=L_{2}$, see also [14]. Throughout, we set

$$
\prod_{j=i}^{k} L_{j}=L_{k} \prod_{j=i}^{k-1} L_{j}, \quad k \geq i, \quad \prod_{j=i}^{k} L_{j}=I, \quad k<i,
$$

that is, the product of operators is defined downwards.

For an initial value problem of the form

$$
u^{\prime}(t)=L u(t)+f(t), \quad t \geq 0, \quad u(0) \text { given },
$$


involving a linear operator $L$ and a time-dependent function $f$, the exact solution can (formally) be represented by the variation-of-constants formula

$$
\begin{aligned}
u(t) & =E_{L}(t) u(0)+\int_{0}^{t} E_{L}(t-\tau) f(\tau) \mathrm{d} \tau, \\
& =E_{L}(t) u(0)+\int_{0}^{t} E_{L}(\tau) f(t-\tau) \mathrm{d} \tau, \quad t \geq 0 .
\end{aligned}
$$

Here, we employ the abbreviation $E_{L}(t)=\mathrm{e}^{t L}, t \geq 0$. Making use of the fact that the first commutator $\left[E_{L_{1}}, L_{2}\right]$ fulfills the following initial value problem

$$
\frac{\mathrm{d}}{\mathrm{d} t}\left[E_{L_{1}}(t), L_{2}\right]=L_{1}\left[E_{L_{1}}(t), L_{2}\right]+\left[L_{1}, L_{2}\right] E_{L_{1}}(t), t \geq 0, \quad\left[E_{L_{1}}(0), L_{2}\right]=0,
$$

the variation-of-constants formula (2.8) implies the relations

$$
\begin{gathered}
{\left[E_{L_{1}}, L_{2}\right]=\mathscr{I}_{+}\left(L_{1}, L_{2}\right) E_{L_{1}}=E_{L_{1}} \mathscr{I}_{-}\left(L_{1}, L_{2}\right),} \\
\mathscr{I}_{ \pm}\left(L_{1}, L_{2}, t\right)=\int_{0}^{t} E_{L_{1}}^{ \pm}(\tau)\left[L_{1}, L_{2}\right] E_{L_{1}}^{\mp}(\tau) \mathrm{d} \tau,
\end{gathered}
$$

where $E_{L}^{ \pm}(t)=E_{L}( \pm t)$; if the variable $t$ is omitted, we write $\mathscr{I}_{ \pm}\left(L_{1}, L_{2}\right)$ for short. Using that

$$
\begin{aligned}
E_{L_{1}} \mathscr{I}_{+}\left(L_{2}, L_{3}\right) & =\mathscr{I}_{+}\left(L_{2}, L_{3}\right) E_{L_{1}}+\left[E_{L_{1}}, \mathscr{I}_{+}\left(L_{2}, L_{3}\right)\right], \\
\mathscr{I}_{-}\left(L_{2}, L_{3}\right) E_{L_{1}} & =E_{L_{1}} \mathscr{I}_{-}\left(L_{2}, L_{3}\right)-\left[E_{L_{1}}, \mathscr{I}_{-}\left(L_{2}, L_{3}\right)\right],
\end{aligned}
$$

an application of the relations in (2.9a) further gives

$$
\begin{aligned}
& E_{L_{1}} \mathscr{I}_{+}\left(L_{2}, L_{3}\right)=\left(\mathscr{I}_{+}\left(L_{2}, L_{3}\right)+\mathscr{I}_{+}\left(L_{1}, \mathscr{I}_{+}\left(L_{2}, L_{3}\right)\right)\right) E_{L_{1}}, \\
& \mathscr{I}_{-}\left(L_{2}, L_{3}\right) E_{L_{1}}=E_{L_{1}}\left(\mathscr{I}_{-}\left(L_{2}, L_{3}\right)-\mathscr{I}_{-}\left(L_{1}, \mathscr{I}_{-}\left(L_{2}, L_{3}\right)\right)\right) .
\end{aligned}
$$

We note that the operator $\mathscr{I}_{ \pm}\left(L_{1}, L_{2}\right)$ is linear with respect to the second argument $L_{2}$.

Let $g$ denote a function that depends on the variable $\tau=\left(\tau_{1}, \tau_{2}, \ldots, \tau_{m}\right) \in \mathbb{R}^{m}$. A Taylor series expansion about $\tau=0$ yields

$$
g(\tau)=\sum_{j=0}^{J} \frac{1}{j !} \partial_{\tau}^{j} g(0) \tau^{j}+\frac{1}{J !} \int_{0}^{1}(1-\zeta)^{J} \partial_{\tau}^{J+1} g(\zeta \tau) \tau^{J+1} \mathrm{~d} \zeta
$$

here, $\partial_{\tau}^{j} g$ denotes the $j$-th derivative of $g$ acting as a multi-linear form on elements in $\mathbb{R}^{j \times m}$.

Throughout, we tacitly suppose that the considered splitting scheme (2.2) fulfills the first-order conditions

$$
\sum_{j=1}^{s} a_{j}=1, \quad \sum_{j=1}^{s} b_{j}=1 .
$$

For $1 \leq j \leq s$ we write $A_{j}=a_{j} A$ and $B_{j}=b_{j} B$ for short and denote

$$
c_{j}=\sum_{i=1}^{j} a_{i}, \quad d_{j}=\sum_{i=1}^{j} b_{i}
$$


moreover, we set $b_{0}=d_{0}=0$ and $B_{0}=0$.

As standard, the Lebesgue space $L^{2}(\Omega)$ of square integrable complex-valued functions $f: \Omega \subset \mathbb{R}^{d} \rightarrow \mathbb{C}$ is endowed with the norm

$$
\|f\|_{L^{2}}^{2}=\int_{\Omega}|f(x)|^{2} \mathrm{~d} x, \quad f \in L^{2}(\Omega) .
$$

The Sobolev space $H^{m}(\Omega)$ comprises all functions with partial derivatives up to order $m \geq 0$ contained in $L^{2}(\Omega)$, where in particular $H^{0}(\Omega)=L^{2}(\Omega)$; the associated norm $\|\cdot\|_{H^{m}}$ is defined through

$$
\|f\|_{H^{m}}^{2}=\sum_{\substack{j=\left(j_{1}, \ldots, j_{d}\right) \in \mathbb{N}^{d} \\ j_{1}+\cdots+j_{d} \leq m}}\left\|\partial^{j} f\right\|_{L^{2}}^{2}, \quad f \in H^{m}(\Omega) .
$$

Detailed information on Sobolev spaces is found in the monograph [1].

We denote by $C$ a generic constant, possibly taking different values at different occurrences.

\section{Local error representation}

In this section, we are concerned with deriving a suitable representation for the local error of an exponential splitting method of the general form (2.2). For this purpose, we interpret the splitting operator $\mathscr{S}$ as exact solution of an initial value problem; rewriting the right-hand side of the differential equation as $(A+B) \mathscr{S}+\mathscr{R}$ and employing the variation-of-constants formula to represent $\mathscr{S}$ yields a relation for the defect operator

$$
\mathscr{L}=\mathscr{S}-\mathscr{E}
$$

that involves the exact solution operator $\mathscr{E}$ of the evolution equation (1.2) and the remainder $\mathscr{R}$. In a recursive procedure, the operator $\mathscr{R}$ is then manipulated further.

For a single term $\mathscr{S}_{j}$ in the splitting operator it holds $\mathscr{S}_{j}^{\prime}=\mathscr{S}_{j} A_{j}+B_{j} \mathscr{S}_{j}$, see (2.2); consequently, the time derivative of $\mathscr{S}$ equals

$$
\mathscr{S}^{\prime}=\sum_{j=1}^{s} \mathscr{S}_{j+1}^{s}\left(\mathscr{S}_{j} A_{j}+B_{j} \mathscr{S}_{j}\right) \mathscr{S}_{1}^{j-1}
$$

We rewrite $\mathscr{S}^{\prime}$ as $(A+B) \mathscr{S}+\mathscr{R}$ and make use of the fact that $\mathscr{S}(0)=I$; this yields the following initial value problem for the splitting operator

$$
\mathscr{S}^{\prime}(t)=(A+B) \mathscr{S}(t)+\mathscr{R}(t), \quad t \geq 0, \quad \mathscr{S}(0)=I
$$

where the operator $\mathscr{R}$ is given through

$$
\mathscr{R}=\sum_{j=1}^{s} \mathscr{S}_{j+1}^{s}\left(\mathscr{S}_{j} A_{j}+B_{j} \mathscr{S}_{j}\right) \mathscr{S}_{1}^{j-1}-(A+B) \mathscr{S} .
$$

An application of the variation-of-constants formula (2.8) implies

$$
\mathscr{S}(t)=\mathscr{E}(t)+\int_{0}^{t} \mathscr{E}(t-\tau) \mathscr{R}(\tau) \mathrm{d} \tau
$$


we therefore obtain the following relation for the defect operator

$$
\mathscr{L}(t)=\int_{0}^{t} \mathscr{E}(t-\tau) \mathscr{R}(\tau) \mathrm{d} \tau,
$$

see (2.1) and (3.1).

Next, we rewrite the operator $\mathscr{R}$ given by (3.2) as $\mathscr{R}=\mathscr{S}_{\sigma+1}^{s} \mathscr{T} \mathscr{S}_{1}^{\sigma}$, where $\sigma=s / 2$ if $s$ is even and $\sigma=(s+1) / 2$ otherwise; this is done in in such a way that the (classical) order conditions of the splitting method (2.2) can be obtained by a straightforward expansion of $\mathscr{T}$. We recall the validity of the first-order conditions (2.11) and that by definition $B_{0}=0$; a simple calculation yields the relation

$$
\mathscr{R}=\sum_{j=1}^{s}\left[\mathscr{S}_{j}^{s}, A_{j}+B_{j-1}\right] \mathscr{S}_{1}^{j-1},
$$

see (2.6) for the definition of the first commutator. Applying the telescopic identity

$$
\left[\mathscr{S}_{j}^{s}, A_{j}+B_{j-1}\right]=\sum_{k=j}^{s} \mathscr{S}_{k+1}^{s}\left[\mathscr{S}_{k}, A_{j}+B_{j-1}\right] \mathscr{S}_{j}^{k-1},
$$

interchanging the order of summation, and using that $\left[\mathscr{S}_{k}, A\right]=\left[E_{B_{k}}, A\right] E_{A_{k}}$ as well as $\left[\mathscr{S}_{k}, B\right]=E_{B_{k}}\left[E_{A_{k}}, B\right]$, further implies

$$
\mathscr{R}=\sum_{k=1}^{s} \mathscr{R}_{k}, \quad \mathscr{R}_{k}=\mathscr{S}_{k+1}^{s}\left(c_{k}\left[E_{B_{k}}, A\right] E_{A_{k}}+d_{k-1} E_{B_{k}}\left[E_{A_{k}}, B\right]\right) \mathscr{I}_{1}^{k-1},
$$

see also (2.12). We first consider $\mathscr{R}_{k}$ for indices $1 \leq k \leq \sigma$; applying the relations in (2.9) that involve $\mathscr{I}_{+}$, we obtain

$$
\begin{gathered}
\mathscr{R}_{k}=\mathscr{S}_{k+1}^{s} C_{k, 0} \mathscr{S}_{1}^{k}, \quad 1 \leq k \leq \sigma \\
C_{k, 0}=c_{k} \mathscr{I}_{+}\left(B_{k}, A\right)+d_{k-1} \mathscr{I}_{+}\left(A_{k}, B\right)+d_{k-1} \mathscr{I}_{+}\left(B_{k}, \mathscr{I}_{+}\left(A_{k}, B\right)\right) .
\end{gathered}
$$

Similarly, for $\sigma+1 \leq k \leq s$ it follows

$$
\begin{gathered}
\mathscr{R}_{k}=\mathscr{S}_{k}^{s} D_{k, 0} \mathscr{S}_{1}^{k-1}, \quad \sigma+1 \leq k \leq s, \\
D_{k, 0}=c_{k} \mathscr{I}_{-}\left(B_{k}, A\right)-c_{k} \mathscr{I}_{-}\left(A_{k}, \mathscr{I}_{-}\left(B_{k}, A\right)\right)+d_{k-1} \mathscr{I}_{-}\left(A_{k}, B\right) .
\end{gathered}
$$

For indices $k=\sigma$ and $k=\sigma+1$, the term $\mathscr{R}_{k}$ already has the desired form; otherwise, for $1 \leq k \leq \sigma-1$ and $\sigma+2 \leq k \leq s$, respectively, we repeatedly apply the identities

$$
\begin{aligned}
\mathscr{S}_{j} C & =\left(C+\mathscr{I}_{+}\left(A_{j}, C\right)+\mathscr{I}_{+}\left(B_{j}, C\right)+\mathscr{I}_{+}\left(B_{j}, \mathscr{I}_{+}\left(A_{j}, C\right)\right)\right) \mathscr{I}_{j}, \\
D \mathscr{S}_{j} & =\mathscr{S}_{j}\left(D-\mathscr{I}_{-}\left(A_{j}, D\right)-\mathscr{I}_{-}\left(B_{j}, D\right)+\mathscr{I}_{-}\left(A_{j}, \mathscr{I}_{-}\left(B_{j}, D\right)\right)\right),
\end{aligned}
$$

that follow from (2.9) for arbitrary operators $C$ and $D$.

Altogether, we finally obtain the following representation of the defect operator. We recall the tacit requirement that the arising unbounded operators are well-defined on suitably chosen domains. 
Theorem 1 (Local error representation) The defect operator $\mathscr{L}=\mathscr{S}-\mathscr{E}$ of the exponential operator splitting method (2.2) possesses the integral representation

$$
\begin{gathered}
\mathscr{L}(t)=\int_{0}^{t} \mathscr{E}(t-\tau) \mathscr{R}(\tau) \mathrm{d} \tau, \quad t \geq 0, \quad \mathscr{R}=\mathscr{S}_{\sigma+1}^{s} \mathscr{T} \mathscr{S}_{1}^{\sigma}, \\
\sigma=\left\{\begin{array}{ll}
\frac{1}{2} s, & \text { s even }, \\
\frac{1}{2}(s+1), & \text { s odd },
\end{array} \quad \mathscr{T}=\sum_{j=0}^{\sigma-1} C_{\sigma-j, j}+\sum_{j=0}^{s-\sigma-1} D_{\sigma+1+j, j} .\right.
\end{gathered}
$$

Here, the quantities $C_{\sigma-j, j}$ and $D_{\sigma+1+j, j}$ are given by recurrence through

$$
\begin{aligned}
C_{k, 0}= & c_{k} \mathscr{I}_{+}\left(B_{k}, A\right)+d_{k-1} \mathscr{I}_{+}\left(A_{k}, B\right)+d_{k-1} \mathscr{I}_{+}\left(B_{k}, \mathscr{I}_{+}\left(A_{k}, B\right)\right), \\
C_{k, j}= & C_{k, j-1}+\mathscr{I}_{+}\left(A_{k+j}, C_{k, j-1}\right)+\mathscr{I}_{+}\left(B_{k+j}, C_{k, j-1}\right) \\
& \quad+\mathscr{I}_{+}\left(B_{k+j}, \mathscr{I}_{+}\left(A_{k+j}, C_{k, j-1}\right)\right), \quad 1 \leq k \leq \sigma, 0 \leq j \leq \sigma-1, \\
D_{k, 0}= & c_{k} \mathscr{I}_{-}\left(B_{k}, A\right)-c_{k} \mathscr{I}_{-}\left(A_{k}, \mathscr{I}_{-}\left(B_{k}, A\right)\right)+d_{k-1} \mathscr{I}_{-}\left(A_{k}, B\right), \\
D_{k, j}= & D_{k, j-1}-\mathscr{I}_{-}\left(A_{k-j}, D_{k, j-1}\right)-\mathscr{I}_{-}\left(B_{k-j}, D_{k, j-1}\right) \\
& \quad+\mathscr{I}_{-}\left(A_{k-j}, \mathscr{I}_{-}\left(B_{k-j}, D_{k, j-1}\right)\right), \quad \sigma+1 \leq k \leq s, \quad 0 \leq j \leq s-\sigma-1,
\end{aligned}
$$

see (2.9) for the definition of $\mathscr{I}_{ \pm}$.

For the Lie-Trotter splitting (2.3a) the above result yields

$$
\mathscr{R}=\mathscr{T} \mathscr{S}_{1}, \quad \mathscr{T}=\mathscr{I}_{+}(B, A) .
$$

On the other hand, for the Strang splitting method (2.4), we obtain

$$
\begin{gathered}
\mathscr{R}=\mathscr{S}_{2} \mathscr{T} \mathscr{S}_{1}, \\
\mathscr{T}=a_{1} \mathscr{I}_{+}\left(B_{1}, A\right)+b_{1} \mathscr{I}_{-}\left(A_{2}, B\right)+\mathscr{I}_{-}\left(B_{2}, A\right)-\mathscr{I}_{-}\left(A_{2}, \mathscr{I}_{-}\left(B_{2}, A\right)\right) ;
\end{gathered}
$$

recall that $c_{2}=a_{1}+a_{2}=1$. For a higher-order scheme, the local error representation of Theorem 1 is investigated in Section 4; applications to linear Schrödinger equations in the semi-classical regime are the contents of Section 5.

\section{Local error expansion and order conditions}

Henceforth, we restrict ourselves to the consideration of an exponential operator splitting method (2.2) of order four involving four compositions, that is, we set $p=s=4$; furthermore, the first-order Lie-Trotter splitting and the second-order Strang splitting are retained as special cases.

\subsection{Expansion of the defect operator}

In the present situation, the afore deduced representation of the defect operator yields $\mathscr{R}=\mathscr{S}_{4} \mathscr{S}_{3} \mathscr{T} \mathscr{S}_{2} \mathscr{S}_{1} ;$ recalling that $\mathscr{I}_{ \pm}$is linear with respect to the second argument 
and that the first order condition (2.11) implies $c_{4}=1$, a brief calculation shows that $\mathscr{T}=\mathscr{T}_{1}+\mathscr{T}_{2}+\mathscr{T}_{3}+\mathscr{T}_{4}$ comprises the following terms

$$
\begin{gathered}
\mathscr{T}_{1}=d_{1} \mathscr{I}_{+}\left(A_{2}, B\right)+c_{1} \mathscr{I}_{+}\left(B_{1}, A\right)+c_{2} \mathscr{I}_{+}\left(B_{2}, A\right) \\
+d_{2} \mathscr{I}_{-}\left(A_{3}, B\right)+d_{3} \mathscr{I}_{-}\left(A_{4}, B\right)+c_{3} \mathscr{I}_{-}\left(B_{3}, A\right)+\mathscr{I}_{-}\left(B_{4}, A\right), \\
\mathscr{T}_{2}=c_{1} \mathscr{I}_{+}\left(A_{2}, \mathscr{I}_{+}\left(B_{1}, A\right)\right)+d_{1} \mathscr{I}_{+}\left(B_{2}, \mathscr{I}_{+}\left(A_{2}, B\right)\right) \\
+c_{1} \mathscr{I}_{+}\left(B_{2}, \mathscr{I}_{+}\left(B_{1}, A\right)\right)-d_{3} \mathscr{I}_{-}\left(A_{3}, \mathscr{I}_{-}\left(A_{4}, B\right)\right) \\
-c_{3} \mathscr{I}_{-}\left(A_{3}, \mathscr{I}_{-}\left(B_{3}, A\right)\right)-\mathscr{I}_{-}\left(A_{3}, \mathscr{I}_{-}\left(B_{4}, A\right)\right) \\
-\mathscr{I}_{-}\left(A_{4}, \mathscr{I}_{-}\left(B_{4}, A\right)\right)-d_{3} \mathscr{I}_{-}\left(B_{3}, \mathscr{I}_{-}\left(A_{4}, B\right)\right) \\
\quad-\mathscr{I}_{-}\left(B_{3}, \mathscr{I}_{-}\left(B_{4}, A\right)\right) \\
\mathscr{T}_{3}=c_{1} \mathscr{I}_{+}\left(B_{2}, \mathscr{I}_{+}\left(A_{2}, \mathscr{I}_{+}\left(B_{1}, A\right)\right)\right)+d_{3} \mathscr{I}_{-}\left(A_{3}, \mathscr{I}_{-}\left(B_{3}, \mathscr{I}_{-}\left(A_{4}, B\right)\right)\right) \\
+\mathscr{I}_{-}\left(A_{3}, \mathscr{I}_{-}\left(A_{4}, \mathscr{I}_{-}\left(B_{4}, A\right)\right)\right)+\mathscr{I}_{-}\left(A_{3}, \mathscr{I}_{-}\left(B_{3}, \mathscr{I}_{-}\left(B_{4}, A\right)\right)\right) \\
+\mathscr{I}_{-}\left(B_{3}, \mathscr{I}_{-}\left(A_{4}, \mathscr{I}_{-}\left(B_{4}, A\right)\right)\right) \\
\mathscr{T}_{4}=-\mathscr{I}_{-}\left(A_{3}, \mathscr{I}_{-}\left(B_{3}, \mathscr{I}_{-}\left(A_{4}, \mathscr{I}_{-}\left(B_{4}, A\right)\right)\right)\right)
\end{gathered}
$$

see also Theorem 1 and (2.12).

We next expand $\mathscr{T}$ with respect to $t$ by means of the auxiliary result (2.10); the (classical) order conditions are then retained from the requirement that all terms in $\mathscr{T}$ involving $t^{q}, 1 \leq q \leq p-1$, should vanish.

At first, we consider multiple compositions of $\mathscr{I}_{ \pm}$. For a family of linear operators $\left(L_{j}\right)_{j}$, we henceforth let

$$
\mathscr{I}_{ \pm}\left(L_{1}, L_{2}, \ldots, L_{m}\right)=\mathscr{I}_{ \pm}\left(L_{1}, \mathscr{I}_{ \pm}\left(L_{2}, \ldots, \mathscr{I}_{ \pm}\left(L_{m-1}, L_{m}\right)\right)\right)
$$

moreover, we employ the standard vector notation $\tau=\left(\tau_{1}, \tau_{2}, \ldots, \tau_{m}\right) \in \mathbb{R}^{m}$ and $\mathrm{d} \tau=\mathrm{d} \tau_{1} \mathrm{~d} \tau_{2} \cdots \mathrm{d} \tau_{m}$. Setting $g=E_{L_{1}}^{ \pm}\left[L_{1}, L_{2}\right] E_{L_{1}}^{\mp}$ and $J=p-2=2$ in (2.10), and integrating with respect to $\tau=\tau_{1} \in[0, t]$ implies

$$
\begin{gathered}
\mathscr{I}_{ \pm}\left(L_{1}, L_{2}, t\right)=\operatorname{ad}_{L_{1}}\left(L_{2}\right) t \pm \frac{1}{2} \operatorname{ad}_{L_{1}}^{2}\left(L_{2}\right) t^{2}+\frac{1}{6} \operatorname{ad}_{L_{1}}^{3}\left(L_{2}\right) t^{3}+R_{1}\left(L_{1}, L_{2}, t\right), \\
R_{1}\left(L_{1}, L_{2}, t\right)= \pm \frac{1}{2} \int_{0}^{t} \int_{0}^{1}(1-\zeta)^{2} r_{1}\left(L_{1}, L_{2}, \zeta \tau\right) \tau^{3} \mathrm{~d} \zeta \mathrm{d} \tau \\
r_{1}\left(L_{1}, L_{2}, \tau\right)=E_{L_{1}}^{ \pm}(\tau) \operatorname{ad}_{L_{1}}^{4}\left(L_{2}\right) E_{L_{1}}^{\mp}(\tau) .
\end{gathered}
$$

Inserting (2.9) into a term of the form $\mathscr{I}_{ \pm}\left(L_{1}, L_{2}, L_{3}\right)$, we obtain

$$
\mathscr{I}_{ \pm}\left(L_{1}, L_{2}, L_{3}, t\right)=\iint_{0}^{t} E_{L_{1}}^{ \pm}\left(\tau_{1}\right) \operatorname{ad}_{L_{1}}\left(E_{L_{2}}^{ \pm}\left(\tau_{2}\right) \operatorname{ad}_{L_{2}}\left(L_{3}\right) E_{L_{2}}^{\mp}\left(\tau_{2}\right)\right) E_{L_{1}}^{\mp}\left(\tau_{1}\right) \mathrm{d} \tau ;
$$


a Taylor series expansions of the integrand further yields

$$
\begin{gathered}
\mathscr{I}_{ \pm}\left(L_{1}, L_{2}, L_{3}, t\right)=\operatorname{ad}_{L_{1}}\left(\operatorname{ad}_{L_{2}}\left(L_{3}\right)\right) t^{2} \pm \frac{1}{2}\left(\operatorname{ad}_{L_{1}}^{2}\left(\operatorname{ad}_{L_{2}}\left(L_{3}\right)\right)\right. \\
\left.+\operatorname{ad}_{L_{1}}\left(\operatorname{ad}_{L_{2}}^{2}\left(L_{3}\right)\right)\right) t^{3}+R_{2}\left(L_{1}, L_{2}, L_{3}, t\right), \\
R_{2}\left(L_{1}, L_{2}, L_{3}, t\right)=\iint_{0}^{t} \int_{0}^{1}(1-\zeta) r_{2}\left(L_{1}, L_{2}, L_{3}, \zeta \tau\right) \mathrm{d} \zeta \mathrm{d} \tau, \\
r_{2}=r_{21} \tau_{1}^{2}+2 r_{22} \tau_{1} \tau_{2}+r_{23} \tau_{2}^{2}, \\
r_{2 j}\left(L_{1}, L_{2}, L_{3}, \tau\right)=E_{L_{1}}^{ \pm}\left(\tau_{1}\right) \operatorname{ad}_{L_{1}}^{4-j}\left(E_{L_{2}}^{ \pm}\left(\tau_{2}\right) \operatorname{ad}_{L_{2}}^{j}\left(L_{3}\right) E_{L_{2}}^{\mp}\left(\tau_{2}\right)\right) E_{L_{1}}^{\mp}\left(\tau_{1}\right) .
\end{gathered}
$$

In a similar manner, by integrating relation (2.10) we obtain

$$
\begin{array}{r}
\mathscr{I}_{ \pm}\left(L_{1}, L_{2}, L_{3}, L_{4}, t\right)=\operatorname{ad}_{L_{1}}\left(\operatorname{ad}_{L_{2}}\left(\operatorname{ad}_{L_{3}}\left(L_{4}\right)\right)\right) t^{3}+R_{3}\left(L_{1}, L_{2}, L_{3}, L_{4}, t\right), \\
R_{3}\left(L_{1}, L_{2}, L_{3}, L_{4}, t\right)=\iiint_{0}^{t} \int_{0}^{1} r_{3}\left(L_{1}, L_{2}, L_{3}, L_{4}, \zeta \tau\right) \mathrm{d} \zeta \mathrm{d} \tau, \\
r_{3}=r_{31} \tau_{1}+r_{32} \tau_{2}+r_{33} \tau_{3}, \\
r_{3 j}\left(L_{1}, L_{2}, L_{3}, L_{4}, \tau\right)=E_{L_{1}}^{ \pm}\left(\tau_{1}\right) \operatorname{ad}_{L_{1}}^{1+\delta_{1 j}}\left(E _ { L _ { 2 } } ^ { \pm } ( \tau _ { 2 } ) \operatorname { a d } _ { L _ { 2 } } ^ { 1 + \delta _ { 2 j } } \left(E_{L_{3}}^{ \pm}\left(\tau_{3}\right)\right.\right. \\
\left.\left.\times \operatorname{ad}_{L_{3}}^{1+\delta_{3 j}}\left(L_{4}\right) E_{L_{3}}^{\mp}\left(\tau_{3}\right)\right) E_{L_{2}}^{\mp}\left(\tau_{2}\right)\right) E_{L_{1}}^{\mp}\left(\tau_{1}\right) ;
\end{array}
$$

here, $\delta_{i j}$ denotes the Kronecker delta. A straightforward application of the defining integral relation for $\mathscr{I}_{ \pm}$gives

$$
\begin{gathered}
\mathscr{I}_{ \pm}\left(L_{1}, L_{2}, L_{3}, L_{4}, L_{5}, t\right)=\iiint \int_{0}^{t} r_{4}\left(L_{1}, L_{2}, L_{3}, L_{4}, L_{5}, \tau\right) \mathrm{d} \tau \\
r_{4}\left(L_{1}, L_{2}, L_{3}, L_{4}, L_{5}, \tau\right)=E_{L_{1}}^{ \pm}\left(\tau_{1}\right) \operatorname{ad}_{L_{1}}\left(E _ { L _ { 2 } } ^ { \pm } ( \tau _ { 2 } ) \operatorname { a d } _ { L _ { 2 } } \left(E_{L_{3}}^{ \pm}\left(\tau_{3}\right)\right.\right. \\
\left.\left.\times \operatorname{ad}_{L_{3}}\left(E_{L_{4}}^{ \pm}\left(\tau_{4}\right) \operatorname{ad}_{L_{4}}\left(L_{5}\right) E_{L_{4}}^{\mp}\left(\tau_{4}\right)\right) E_{L_{3}}^{\mp}\left(\tau_{3}\right)\right) E_{L_{2}}^{\mp}\left(\tau_{2}\right)\right) E_{L_{1}}^{\mp}\left(\tau_{1}\right),
\end{gathered}
$$

see (2.9).

\subsection{Order conditions}

We next insert the above expansions (4.2) into (4.1). For $1 \leq q \leq p-1=3$, we then collect all expressions involving $t^{q}$ which corresponds to the term of order $q+1$ in the defect operator, see Theorem 1; thereby, we make use of the fact that the iterated commutators $\operatorname{ad}_{A}^{j}(B), \operatorname{ad}_{B}^{j}(A), 1 \leq j \leq 3$, and $\operatorname{ad}_{A}\left(\operatorname{ad}_{B}\left(\operatorname{ad}_{A}(B)\right)\right)$ are independent.

We first determine all terms in $\mathscr{T}_{1}$ that involve $t$, see (4.1) and (4.2a); this yields the second-order condition

$$
a_{2} d_{1}+a_{3} d_{2}+a_{4} d_{3}-b_{1} c_{1}-b_{2} c_{2}-b_{3} c_{3}-b_{4}=0 .
$$

Collecting the terms in $\mathscr{T}_{1}$ and $\mathscr{T}_{2}$ that comprise $t^{2}$, the third-order conditions

$$
\begin{gathered}
\frac{1}{2} a_{2}^{2} d_{1}-\frac{1}{2} a_{3}^{2} d_{2}-\frac{1}{2} a_{4}^{2} d_{3}-a_{3} a_{4} d_{3}-a_{2} b_{1} c_{1}+a_{3} b_{3} c_{3}+a_{3} b_{4}+a_{4} b_{4}=0, \\
\frac{1}{2} b_{1}^{2} c_{1}+\frac{1}{2} b_{2}^{2} c_{2}-\frac{1}{2} b_{3}^{2} c_{3}-\frac{1}{2} b_{4}^{2}+b_{1} b_{2} c_{1}-b_{3} b_{4}-a_{2} b_{2} d_{1}+a_{4} b_{3} d_{3}=0,
\end{gathered}
$$


follow, see (4.2a)-(4.2b). Finally, we consider all terms in $\mathscr{T}_{1}, \mathscr{T}_{2}$, and $\mathscr{T}_{3}$ that involve $t^{3}$ and obtain the fourth-order conditions

$$
\begin{aligned}
& \frac{1}{6} a_{2}^{3} d_{1}+\frac{1}{6} a_{3}^{3} d_{2}+\frac{1}{6} a_{4}^{3} d_{3}-\frac{1}{2} a_{2}^{2} b_{1} c_{1}+\frac{1}{2} a_{3}^{2} a_{4} d_{3}+\frac{1}{2} a_{3} a_{4}^{2} d_{3}-\frac{1}{2} a_{3}^{2} b_{3} c_{3} \\
& \quad-\frac{1}{2} a_{3}^{2} b_{4}-\frac{1}{2} a_{4}^{2} b_{4}-a_{3} a_{4} b_{4}=0 \\
& \frac{1}{6} b_{1}^{3} c_{1}+\frac{1}{6} b_{2}^{3} c_{2}+\frac{1}{6} b_{3}^{3} c_{3}+\frac{1}{6} b_{4}^{3}-\frac{1}{2} a_{2} b_{2}^{2} d_{1}+\frac{1}{2} b_{1} b_{2}^{2} c_{1}+\frac{1}{2} b_{1}^{2} b_{2} c_{1} \\
& \quad-\frac{1}{2} a_{4} b_{3}^{2} d_{3}+\frac{1}{2} b_{3}^{2} b_{4}+\frac{1}{2} b_{3} b_{4}^{2}=0 \\
& -\frac{1}{2} a_{2} b_{1}^{2} c_{1}-\frac{1}{2} a_{3} b_{3}^{2} c_{3}-\frac{1}{2} a_{3} b_{4}^{2}-\frac{1}{2} a_{4} b_{4}^{2}+\frac{1}{2} a_{2}^{2} b_{2} d_{1}+\frac{1}{2} a_{4}^{2} b_{3} d_{3}-a_{2} b_{1} b_{2} c_{1} \\
& \quad+a_{3} a_{4} b_{3} d_{3}-a_{3} b_{3} b_{4}-a_{4} b_{3} b_{4}=0
\end{aligned}
$$

see $(4.2 \mathrm{a})-(4.2 \mathrm{c})$.

It is notable that the order conditions (2.11) and (4.3) form a system of 8 nonlinear algebraic equations in 8 unknowns, whereas the approach used in [26] yields a system of 16 (redundant) polynomial equations. Resolving the above order conditions under the additional symmetry requirements $a_{1}=0, a_{4}=a_{2}, b_{3}=b_{2}, b_{4}=b_{1}$, we retain the fourth-order splitting scheme (2.5). The Lie-Trotter splitting method (2.3a) follows at once from the first-order condition (2.11) with $s=1$; the Strang splitting scheme (2.4) is obtained from (2.11) and (4.3a) by setting $s=2, a_{j}=b_{j}=0, j=3,4$, and furthermore $a_{1}=0$ or $b_{2}=0$, respectively.

\section{Linear Schrödinger equations in the semi-classical regime}

In this section, we demonstrate the ability of our approach to provide optimal error estimates for time-splitting methods when applied to linear Schrödinger equations in the semi-classical regime; for simplicity, we restrict ourselves to a single space dimension and give the theoretical details for the first-order Lie-Trotter splitting method and the second-order Strang splitting method only.

\subsection{Error estimates}

In the following, we consider the time-dependent Schrödinger equation (1.1), subject to periodic boundary conditions on a bounded interval $\Omega \subset \mathbb{R}$, with $U: \Omega \rightarrow \mathbb{R}$ a sufficiently smooth periodic function. To cast (1.1) into the abstract form (1.2) and to capture the dependence on the critical parameter, we let

$$
A=\varepsilon \hat{A}, \quad \hat{A}=\mathrm{i} \partial_{x}^{2}, \quad B=\hat{B} / \varepsilon, \quad \hat{B}=-\mathrm{i} U
$$

By Stone's Theorem, see ENGEL AND NAGEL [11], e.g., for any parameter value $\varepsilon>$ 0 , the (unbounded) differential operator $A$ and the multiplication operator $B$ generate unitary evolution operators on $L^{2}(\Omega)$, that is, the relations

$$
\left\|\mathrm{e}^{t A}\right\|_{L^{2} \leftarrow L^{2}}=1, \quad\left\|\mathrm{e}^{t B}\right\|_{L^{2} \leftarrow L^{2}}=1, \quad t \in \mathbb{R},
$$

are valid; moreover, the exact solution operator $\mathrm{e}^{t(A+B)}$ is unitary on $L^{2}(\Omega)$. 
Lie-Trotter splitting. Applying the Lie-Trotter splitting method (2.3a), which is of (classical) order one, yields approximations to the exact solution through

$$
u_{n+1}=\mathrm{e}^{h_{n} B} \mathrm{e}^{h_{n} A} u_{n} \approx u\left(t_{n+1}\right)=\mathrm{e}^{h_{n}(A+B)} u\left(t_{n}\right), \quad 0 \leq n \leq N-1,
$$

see also (2.2). In the present situation, our local error representation results in

$$
\begin{aligned}
\mathscr{L}\left(h_{n}\right) u\left(t_{n}\right) & =\left(\mathrm{e}^{h_{n} B} \mathrm{e}^{h_{n} A}-\mathrm{e}^{h_{n}(A+B)}\right) u\left(t_{n}\right) \\
& =\int_{0}^{h_{n}} \int_{0}^{\tau_{1}} \mathrm{e}^{\left(h_{n}-\tau_{1}\right)(A+B)} \mathrm{e}^{\tau_{2} B}[B, A] \mathrm{e}^{\left(\tau_{1}-\tau_{2}\right) B} \mathrm{e}^{\tau_{1} A} u\left(t_{n}\right) \mathrm{d} \tau_{2} \mathrm{~d} \tau_{1},
\end{aligned}
$$

see Theorem 1 and (3.5a); that is, the local error is of order two with respect to the time stepsize $h_{n}$, provided that the involved evolution operators and the exact solution remain bounded in suitably chosen function spaces. More precisely, due to the fact that for $(5.1)$

$$
[A, B]=[\varepsilon \hat{A}, \hat{B} / \varepsilon]=\left[\partial_{x}^{2}, U\right]=2 \partial_{x} U \partial_{x}+\partial_{x}^{2} U
$$

i.e., $[A, B]$ is a first-order differential operator with coefficients involving the first and second spatial derivatives of $U$, it follows

$$
\|[A, B] u\|_{L^{2}} \leq C\left(\partial_{x}^{2} U\right)\|u\|_{H^{1}}
$$

where $C\left(\partial_{x}^{2} U\right)=\max \left\{2\left\|\partial_{x} U\right\|_{L^{\infty}},\left\|\partial_{x}^{2} U\right\|_{L^{\infty}}\right\}$, see also (2.13). Employing the stability bounds (5.2), we obtain the estimate

$$
\begin{aligned}
\left\|\mathscr{L}\left(h_{n}\right) u\left(t_{n}\right)\right\|_{L^{2}} & \leq C\left(\partial_{x}^{2} U\right) \int_{0}^{h_{n}} \int_{0}^{\tau_{1}}\left\|\mathrm{e}^{-\mathrm{i}\left(\tau_{1}-\tau_{2}\right) U / \varepsilon} \mathrm{e}^{\mathrm{i} \varepsilon \tau_{1} \partial_{x}^{2}} u\left(t_{n}\right)\right\|_{H^{1}} \mathrm{~d} \tau_{2} \mathrm{~d} \tau_{1} \\
& \leq C\left(\partial_{x}^{2} U\right) \frac{h_{n}^{2}}{\varepsilon}\left(h_{n}\left\|\partial_{x} U\right\|_{L^{\infty}}\left\|u\left(t_{n}\right)\right\|_{L^{2}}+\varepsilon\left\|u\left(t_{n}\right)\right\|_{H^{1}}\right) .
\end{aligned}
$$

We note that the first spatial derivative $\chi=\partial_{x} \psi$ of the exact solution to (1.1) fulfills the differential equation

$$
\text { i } \varepsilon \partial_{t} \chi(x, t)=-\varepsilon^{2} \partial_{x}^{2} \chi(x, t)+U(x) \chi(x, t)+\partial_{x} U(x) \psi(x, t), \quad x \in \mathbb{R}, \quad t \geq 0,
$$

that is, in abstract form, with $v(t)=\chi(\cdot, t)$ we have

$$
v^{\prime}(t)=(A+B) v(t)+\partial_{x} B u(t), \quad t \geq 0, \quad v(0) \text { given } .
$$

Consequently, by means of the variation-of-constants formula (2.8) it follows

$$
\varepsilon\|u(t)\|_{H^{1}} \leq \sqrt{2} \varepsilon\|u(0)\|_{H^{1}}+t\left\|\partial_{x} U\right\|_{L^{\infty}}\|u(0)\|_{L^{2}}, \quad t \geq 0 .
$$

A standard Lady Windermere's Fan argument

$$
u_{N}-u\left(t_{N}\right)=\prod_{j=0}^{N-1} \mathscr{S}\left(h_{j}\right)\left(u_{0}-u(0)\right)+\sum_{n=0}^{N-1} \prod_{j=n+1}^{N-1} \mathscr{S}\left(h_{j}\right) \mathscr{L}\left(h_{n}\right) u\left(t_{n}\right)
$$


together with the stability bounds in (5.2) thus yields

$$
\begin{gathered}
\left\|u_{N}-u\left(t_{N}\right)\right\|_{L^{2}} \leq\left\|u_{0}-u(0)\right\|_{L^{2}}+\sum_{n=0}^{N-1}\left\|\mathscr{L}\left(h_{n}\right) u\left(t_{n}\right)\right\|_{L^{2}} \\
\leq\left\|u_{0}-u(0)\right\|_{L^{2}} \\
\quad+C\left(\partial_{x}^{2} U\right) \sum_{n=0}^{N-1} \frac{h_{n}^{2}}{\varepsilon}\left(h_{n}\left\|\partial_{x} U\right\|_{L^{\infty}}\left\|u\left(t_{n}\right)\right\|_{L^{2}}+\varepsilon\left\|u\left(t_{n}\right)\right\|_{H^{1}}\right) \\
\leq\left\|u_{0}-u(0)\right\|_{L^{2}} \\
+C\left(\partial_{x}^{2} U\right) \sum_{n=0}^{N-1} \frac{h_{n}^{2}}{\varepsilon}\left(\left(h_{n}+t_{n}\right)\left\|\partial_{x} U\right\|_{L^{\infty}}\|u(0)\|_{L^{2}}\right. \\
\left.+\sqrt{2} \varepsilon\|u(0)\|_{H^{1}}\right) .
\end{gathered}
$$

Altogether, we obtain the following convergence result.

Theorem 2 (Global error estimate, Lie-Trotter splitting) Suppose that the potential $U: \Omega \rightarrow \mathbb{R}$ is twice differentiable with bounded derivatives and that the initial value $u(0)$ remains bounded in $H^{1}(\Omega)$. Then, the Lie-Trotter splitting method (2.3a) applied to the time-dependent linear Schrödinger equation (1.1) fulfills the global error estimate

$$
\begin{aligned}
\left\|u_{N}-u\left(t_{N}\right)\right\|_{L^{2}} \leq & \left\|u_{0}-u(0)\right\|_{L^{2}} \\
& +C\left(\partial_{x}^{2} U\right) \sum_{n=0}^{N-1} \frac{h_{n}^{2}}{\varepsilon}\left(t_{n}\left\|\partial_{x} U\right\|_{L^{\infty}}\|u(0)\|_{L^{2}}+\varepsilon\|u(0)\|_{H^{1}}\right)
\end{aligned}
$$

with constant $C\left(\partial_{x}^{2} U\right)=2 \max \left\{2\left\|\partial_{x} U\right\|_{L^{\infty}},\left\|\partial_{x}^{2} U\right\|_{L^{\infty}}\right\}$

Especially, for classical Wentzel-Kramers-Brillouin (WKB) initial values where the quantity $\varepsilon^{j}\|u(0)\|_{H^{j}}$ remains bounded for $j=1$, that is, it holds

$$
\varepsilon^{j}\|u(0)\|_{H^{j}} \leq M_{j}
$$

for a constant $M_{j}>0$, see [2], e.g., the bound

$$
\left\|u_{N}-u\left(t_{N}\right)\right\|_{L^{2}} \leq\left\|u_{0}-u(0)\right\|_{L^{2}}+C \frac{h}{\varepsilon}
$$

follows with maximum time step $h=\max \left\{h_{n}: 0 \leq n \leq N-1\right\}$ and constant $C$ depending on $M_{j}, j=0,1,\left\|\partial_{x}^{j} U\right\|_{L^{\infty}}, j=1,2$, and $t_{N}$. 
Strang splitting. For the second-order Strang splitting method (2.4), relation (3.5b) and an expansion in the lines of Section 4.1 yields

$$
\begin{aligned}
& \mathscr{L}\left(h_{n}\right) u\left(t_{n}\right)= \int_{0}^{h_{n}} \int_{0}^{\tau_{1}} \mathrm{e}^{\left(h_{n}-\tau_{1}\right)(A+B)} \mathrm{e}^{\tau_{1} b_{2} B} \mathrm{e}^{\tau_{1} a_{2} A} \\
& \times\left(\int_{0}^{\tau_{1}} a_{2} b_{2} \mathrm{e}^{-\tau_{2} a_{2} A}\left[A, \mathrm{e}^{-\tau_{3} b_{2} B}[A, B] \mathrm{e}^{\tau_{3} b_{2} B}\right] \mathrm{e}^{\tau_{2} a_{2} A} \mathrm{~d} \tau_{3}\right. \\
&+\int_{0}^{1} \tau_{2}\left(-a_{2}^{2} b_{1} \mathrm{e}^{-\zeta \tau_{2} a_{2} A}[A,[A, B]] \mathrm{e}^{\zeta \tau_{2} a_{2} A}\right. \\
&+a_{1} b_{1}^{2} \mathrm{e}^{\zeta \tau_{2} b_{1} B}[B,[B, A]] \mathrm{e}^{-\zeta \tau_{2} b_{1} B} \\
&\left.\left.-b_{2}^{2} \mathrm{e}^{-\zeta \tau_{2} b_{2} B}[B,[B, A]] \mathrm{e}^{\zeta \tau_{2} b_{2} B}\right) \mathrm{~d} \zeta\right) \\
& \times \mathrm{e}^{\tau_{1} b_{1} B} \mathrm{e}^{\tau_{1} a_{1} A} u\left(t_{n}\right) \mathrm{d} \tau_{2} \mathrm{~d} \tau_{1}
\end{aligned}
$$

see Theorem 1; we note that in $\mathscr{T}$ the first order term $\left(\left(a_{2}-a_{1}\right) b_{1}-b_{2}\right) \operatorname{ad}_{A}(B)$ vanishes due to the validity of the order conditions (2.11) and (4.3a). In particular, we have $\mathscr{L}\left(h_{n}\right) u\left(t_{n}\right)=\mathscr{O}\left(h_{n}^{3}\right)$, whenever the involved quantities are bounded in suitably chosen function spaces. Extending the previous considerations for the Lie-splitting method, we obtain

$$
\begin{gathered}
\|[B,[A, B]] u\|_{L^{2}} \leq C\left(\partial_{x} U\right) \frac{1}{\varepsilon}\|u\|_{L^{2}}, \\
\left\|\operatorname{ad}_{A}^{j}(B) u\right\|_{L^{2}} \leq C\left(\partial_{x}^{2 j} U\right) \varepsilon^{j-1}\|u\|_{H^{j}}, \quad j=1,2, \\
\left\|\left[A, \mathrm{e}^{-t B}[A, B] \mathrm{e}^{t B}\right] u\right\|_{L^{2}} \leq C\left(\partial_{x}^{4} U\right)\left(\varepsilon\|u\|_{H^{2}}+|t|\|u\|_{H^{1}}\right), \\
\left\|\mathrm{e}^{\tau_{1} a_{2} A} \mathscr{S}_{1}(\tau) u\right\|_{H^{j}} \leq C\left(\partial_{x}^{j} U\right) \sum_{\ell=0}^{j} \frac{|\tau|^{j-\ell}}{\varepsilon^{j-\ell}}\|u\|_{H^{\ell}}, \quad j=1,2,
\end{gathered}
$$

see also (5.4); we indicate the dependence of the constants on the highest space derivative of $U$. With the help of the variation-of-constants formula (2.8) and (5.5) the estimate

$$
\begin{aligned}
\varepsilon^{2}\|u(t)\|_{H^{2}} \leq C & \varepsilon^{2}\|u(0)\|_{H^{2}} \\
& +C\left(\partial_{x}^{2} U\right) \varepsilon t\|u(0)\|_{H^{1}}+C\left(\partial_{x} U\right) t^{2}\|u(0)\|_{L^{2}}, \quad t \geq 0
\end{aligned}
$$

follows; the constant $C$ arises in the estimation by the $H^{2}$-Sobolev norm. Consequently, by means of a Lady Windermere's Fan (5.6) argument and the stability bounds (5.2), we obtain the following result; in the present situation, a simple (but more tedious) calculation in the lines of the Lie-splitting method would yield the precise form of the involved constant.

Theorem 3 (Global error estimate, Strang splitting) Suppose that $U: \Omega \rightarrow \mathbb{R}$ is four times differentiable with bounded derivatives and that the initial value $u(0)$ remains bounded in $H^{2}(\Omega)$. Then, the Strang splitting method (2.4) applied to the 
time-dependent linear Schrödinger equation (1.1) fulfills the global error estimate

$$
\begin{aligned}
\left\|u_{N}-u\left(t_{N}\right)\right\|_{L^{2}} \leq \| & u_{0}-u(0) \|_{L^{2}} \\
& +C \sum_{n=0}^{N-1} \frac{h_{n}^{3}}{\varepsilon}\left(\|u(0)\|_{L^{2}}+\varepsilon\|u(0)\|_{H^{1}}+\varepsilon^{2}\|u(0)\|_{H^{2}}\right)
\end{aligned}
$$

with constant $C>0$ depending on $\left\|\partial_{x}^{j} U\right\|_{L^{\infty}}, 1 \leq j \leq 4$, and $t_{N}$.

For classical WKB initial values satisfying (5.7) for $j=1,2$, it follows

$$
\left\|u_{N}-u\left(t_{N}\right)\right\|_{L^{2}} \leq\left\|u_{0}-u(0)\right\|_{L^{2}}+C \frac{h^{2}}{\varepsilon}
$$

with $h=\max \left\{h_{n}: 0 \leq n \leq N-1\right\}$ and constant $C$ depending on $M_{j}, 0 \leq j \leq 2$, $\left\|\partial_{x}^{j} U\right\|_{L^{\infty}}, 1 \leq j \leq 4$, and $t_{N}$. For small parameter values $0<\varepsilon<<1$, the above estimates (5.8) and (5.11) show that for the Strang-splitting method the stepsize restriction $h$ sufficiently smaller than $\sqrt{\varepsilon}$ is weaker than for the first-order Lie-Trotter splitting, where time steps sufficiently smaller than $\varepsilon$ have to be required.

Higher-order splitting. Extending the above considerations to a splitting method of (classical) order $p$ yields the following convergence result.

Theorem 4 (Global error estimate) Assume that the exponential operator splitting method (2.2) satisfies the (classical) pth-order conditions for $p \geq 1$. Suppose further that the potential $U: \Omega \rightarrow \mathbb{R}$ is $2 p$ times differentiable with bounded derivatives and that the initial value $u(0)$ remains bounded in $H^{p}(\Omega)$. Then, the splitting method applied to the time-dependent linear Schrödinger equation (1.1) fulfills the global error estimate

$$
\left\|u_{N}-u\left(t_{N}\right)\right\|_{L^{2}} \leq\left\|u_{0}-u(0)\right\|_{L^{2}}+C \sum_{n=0}^{N-1} \frac{h_{n}^{p+1}}{\varepsilon} \sum_{j=0}^{p} \varepsilon^{j}\|u(0)\|_{H^{j}}
$$

here, the constant $C>0$ depends on $\left\|\partial_{x}^{j} U\right\|_{L^{\infty}}, 0 \leq j \leq 2 p$, and the end time $t_{N}$.

Proof As a detailed proof of Theorem 4 would involve several technicalities, we only indicate the main ingredients.

From the local error representation given in Theorem 1 and a further expansion of the defect in the lines of Section 4.1, we conclude that for a splitting method of order $p$ the local error $\mathscr{L}\left(h_{n}\right) u\left(t_{n}\right)$ comprises the $p$-th iterated commutator of (certain combinations of) $A$ and $B$ as well as exponentials of $A$ and $B$. Note that in the present situation, the iterated commutator $\operatorname{ad}_{A}^{p}(B)$ is a differential operator of order $p$ involving $\varepsilon^{p-1}$ and as highest derivative $\partial_{x}^{2 p} U$; on the other hand, the iterated commutator $\operatorname{ad}_{B}^{j}(A)$ vanishes for $j \geq 3$. Disregarding for simplicity the precise form of the arising constants, it suffices to estimate the principal error terms. For this purpose, it is essential to extend the auxiliary bounds (5.9) and (5.10), proceeding by induction. 


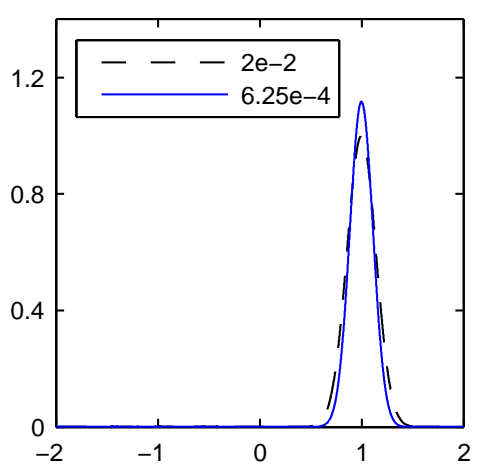

Fig. 5.1 Numerical solution $|\psi(x, 0.64)|$ of the linear Schrödinger equation (5.13) for two different values of the critical parameter $\varepsilon$.

As a consequence, for WKB initial values satisfying (5.7) for $1 \leq j \leq p$, we obtain

$$
\left\|u_{N}-u\left(t_{N}\right)\right\|_{L^{2}} \leq\left\|u_{0}-u(0)\right\|_{L^{2}}+C \frac{h^{p}}{\varepsilon}, \quad h=\max _{0 \leq n \leq N-1} h_{n},
$$

with constant $C>0$ depending on $M_{j}, 0 \leq j \leq p,\left\|\partial_{x}^{j} U\right\|_{L^{\infty}}, 1 \leq j \leq 2 p$, and $t_{N}$. From this error estimate we conclude that higher-order exponential operator splitting methods are favourable for the time-integration of linear Schrödinger equations in the semi-classical regime, provided that the time stepsize fulfills the requirement $h$ sufficiently smaller than $\sqrt[p]{\varepsilon}$.

\subsection{Numerical example}

Following the numerical observations of [3, Example 3] for the Strang splitting, we next illustrate the optimality of the error estimate (5.12) with respect to the critical parameter $0<\varepsilon<<1$ for the first-order Lie-splitting method (2.3a), the second-order Strang splitting method (2.4), and the fourth-order splitting method (2.5) by Yoshida, when applied to the time-dependent linear Schrödinger equation

$$
\begin{gathered}
\text { i } \varepsilon \partial_{t} \psi(x, t)=-\frac{1}{2} \varepsilon^{2} \Delta \psi(x, t)+\frac{1}{2} x^{2} \psi(x, t), \\
\psi(x, 0)=\mathrm{e}^{-25(x-1 / 2)^{2}} \mathrm{e}^{\mathrm{i}(1+x) / \varepsilon}, \quad-2 \leq x \leq 2, \quad 0 \leq t \leq 0.64,
\end{gathered}
$$

subject to periodic boundary conditions. The values of the critical parameter $\varepsilon$ are chosen in the range $6.25 \cdot 10^{-4}$ to $4 \cdot 10^{-2}$. The problem in discretised in space by the Fourier-spectral method with $M$ basis functions. In order to determine the global error in the time integration, for stepsizes $h$ ranging from $1.5625 \cdot 10^{-4}$ to $4 \cdot 10^{-2}$, a reference solution is computed by the fourth-order Runge-Kutta-Nyström splitting method proposed in [6] with time stepsize $h \cdot 10^{-1}$.

In Figure 5.1, the shape of the numerical solution at the final time is illustrated for two different values of the critical parameter. In Figure 5.2, the global temporal 


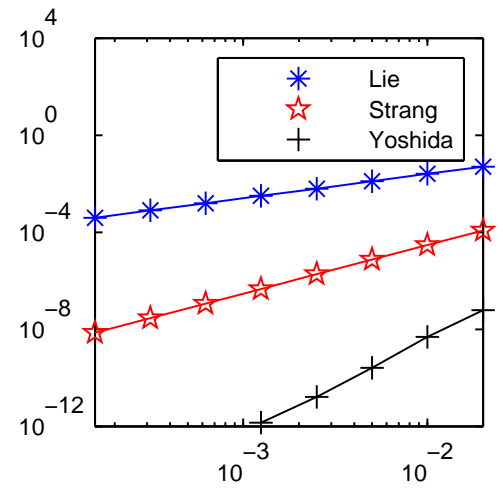

Fig. 5.2 Numerical convergence orders of different time-splitting methods (global error versus stepsize).

errors versus the time stepsizes are displayed for $\varepsilon=4 \cdot 10^{-2}$ and $M=256$, showing the expected convergence orders of the time-splitting methods.

\begin{tabular}{|c|c|c|}
\hline critical parameter & global error $(p=1)$ & ratio $(p=1)$ \\
\hline \hline 0.020000 & $1.908313855543586 \mathrm{e}-001$ & $-9.546799767444528 \mathrm{e}-001$ \\
\hline 0.010000 & $3.698597849512038 \mathrm{e}-001$ & $-8.876216181670132 \mathrm{e}-001$ \\
\hline 0.005000 & $6.842862992889865 \mathrm{e}-001$ & $-5.347591837576926 \mathrm{e}-001$ \\
\hline 0.002500 & $9.913257778242306 \mathrm{e}-001$ & \\
\hline \hline critical parameter & global error $(p=1, h / 4)$ & ratio $(p=1, h / 4)$ \\
\hline \hline 0.020000 & $4.759083334913000 \mathrm{e}-002$ & $-9.795186767037100 \mathrm{e}-001$ \\
\hline 0.010000 & $9.383995971552103 \mathrm{e}-002$ & $-9.887465227244113 \mathrm{e}-001$ \\
\hline 0.005000 & $1.862216516131053 \mathrm{e}-001$ & $-9.728227523631063 \mathrm{e}-001$ \\
\hline 0.002500 & $3.654929489758708 \mathrm{e}-001$ & $-8.940389596804734 \mathrm{e}-001$ \\
\hline 0.001250 & $6.792216918020555 \mathrm{e}-001$ & $-5.448625017980767 \mathrm{e}-001$ \\
\hline 0.000625 & $9.909038225216962 \mathrm{e}-001$ & \\
\hline \hline critical parameter & global error $(p=2)$ & ratio $(p=2)$ \\
\hline \hline 0.020000 & $7.729399568231682 \mathrm{e}-004$ & $-9.118303667208469 \mathrm{e}-001$ \\
\hline 0.010000 & $1.454233181843335 \mathrm{e}-003$ & $-9.763859382146316 \mathrm{e}-001$ \\
\hline 0.005000 & $2.861248016688788 \mathrm{e}-003$ & $-9.939800245764093 \mathrm{e}-001$ \\
\hline 0.002500 & $5.698667358149775 \mathrm{e}-003$ & $-9.984569484384943 \mathrm{e}-001$ \\
\hline 0.001250 & $1.138515107875544 \mathrm{e}-002$ & $-9.994894832381450 \mathrm{e}-001$ \\
\hline 0.000625 & $2.276224600976379 \mathrm{e}-002$ & \\
\hline \hline critical parameter & global error $(p=4)$ & ratio $(p=4)$ \\
\hline \hline 0.020000 & $1.760514889939917 \mathrm{e}-006$ & $-9.667658211629046 \mathrm{e}-001$ \\
\hline 0.010000 & $3.440845825158100 \mathrm{e}-006$ & $-9.914955815231308 \mathrm{e}-001$ \\
\hline 0.005000 & $6.841244690937256 \mathrm{e}-006$ & $-9.978612083014881 \mathrm{e}-001$ \\
\hline 0.002500 & $1.366222015470383 \mathrm{e}-005$ & $-9.994645019027982 \mathrm{e}-001$ \\
\hline 0.001250 & $2.731429993314372 \mathrm{e}-005$ & $-9.998660746803390 \mathrm{e}-001$ \\
\hline 0.000625 & $5.462352893104207 \mathrm{e}-005$ & \\
\hline
\end{tabular}

Table 5.1 Dependence on the critical parameter. Global error and ratio for the Lie-splitting method (top, $p=1$ ), the Strang-splitting method (middle, $p=2$ ), and the splitting method by Yoshida (bottom, $p=4$ ). 
In Table 5.1, the global temporal errors and the corresponding ratios are displayed; that is, for a fixed time stepsize $h$ and different parameter values $\varepsilon$ we determine the global temporal error $\operatorname{err}(\varepsilon)$ and the corresponding ratios

$$
\operatorname{ratio}(\varepsilon)=\log \left(\frac{\operatorname{err}(\varepsilon)}{\operatorname{err}(\varepsilon / 2)}\right) / \log (2)
$$

From the convergence bound given in Theorem 4 and the resulting global error estimate (5.12) we expect the ratios to approach the value -1 . For the Strang splitting method and the splitting method by Yoshida, we set $h=4 \cdot 10^{-2}$ and $M=4096$, whereas for the Lie-splitting method a reduced time step $h / 4$ is required for smaller values of $\varepsilon$. The results in Table 5.1 confirm the expected dependence $\mathscr{O}(1 / \varepsilon)$ of the temporal global error on the critical parameter.

\section{Conclusions}

In this paper, we were concerned with deducing a local error representation for exponential operator splitting methods applied to evolutionary problems that involve critical parameters. To illustrate the general mechanism, we focused on the first-order Lie splitting method, the second-order Strang splitting method, and a fourth-order splitting method by Yoshida.

In particular, our analytical framework applies to Schrödinger equations in the semi-classical regime with critical parameter $0<\varepsilon<<1$. For WKB initial values it is concluded that higher-order exponential operator splitting methods are favourable under the time stepsize restriction $h$ sufficiently smaller than $\sqrt[p]{\varepsilon}$; the dependence of the global temporal error on the time stepsize and the critical parameter is confirmed in a numerical example.

\section{Acknowledgement}

We wish to dedicate this work to Michelle Schatzman on the occasion of her 60th birthday. Further, we thank the anonymous referees for valuable comments. This work was supported by the Austrian Science Fund (FWF) under project P21620-N13 and the Frankreich-Schwerpunkt - Le Pôle interdisciplinaire d'études françaises of the University of Innsbruck.

\section{References}

1. R.A. Adams. Sobolev Spaces. Academic Press, San Diego, CA, 1978.

2. W. BAO, D. JAKSCH, AND P. MARKOWICH. Numerical solution of the Gross-Pitaevskii equation for Bose-Einstein condensation. J. Comp. Phys. 187 (2003), 318-342.

3. W. BAO, S. JIN, AND P. MARKOWICH. On time-splitting spectral approximations for the Schrödinger equation in the semiclassical regime. J. Comput. Phys. 175 (2002), 487-524. 
4. W. BAO, S. JIN, AND P. MARKOWICH. Numerical study of time-splitting spectral discretisations of nonlinear Schrödinger equations in the semiclassical regimes. SIAM J. Sci. Comput. 25/1 (2003), 27-64.

5. W. BAO AND J. SHEN. A fourth-order time-splitting Laguerre-Hermite pseudospectral method for Bose-Einstein condensates. SIAM J. Sci. Comput. 26/6 (2005), 2010-2028.

6. S. Blanes And P.C. Moan. Practical Symplectic Partitioned Runge-Kutta and Runge-KuttaNyström Methods. J. Comput. Appl. Math. 142 (2002), 313-330.

7. M. Caliari, Ch. Neuhauser, And M. Thalhammer. High-order time-splitting Hermite and Fourier spectral methods for the Gross-Pitaevskii equation. J. Comput. Phys. 228 (2009), 822-832.

8. F. Castella, Ph. Chartier, S. Descombes, and G. Vilmart. Splitting methods with complex times for parabolic equations. BIT Numer. Math. 49/3 (2009), 487-508.

9. S. Descombes, T. Dumont, V. Louvet, And M. Massot. On the local and global errors of splitting approximations of reaction-diffusion equations with high spatial gradients. Intern. $\mathrm{J}$. Computer Math, 84/6 (2007), 749-765.

10. S. Descombes And M. Schatzman. Strang's formula for holomorphic semi-groups. J. Math. Pures Appl. 81 (2002), 93-114.

11. K.J. ENGEL AND R. NAGEL. One-Parameter Semigroups for Linear Evolution Equations. Springer, New York, 2000.

12. E. FaOU, V. Gradinaru, And CH. Lubich. Computing semi-classical quantum dynamics with Hagedorn wavepackets. SIAM J. Sci. Comput. $31 / 4$ (2009), 3027-3041.

13. L. GAUCKLER. Convergence of a split-step Hermite method for the Gross-Pitaevskii equation. IMA J. Numer. Anal., 2010 (published online), doi:10.1093/imanum/drp041.

14. E. Hairer, Ch. Lubich, And G. WAnNer. Geometric Numerical Integration. StructurePreserving Algorithms for Ordinary Differential Equations. Springer, Berlin, 2002.

15. E. HANSEN AND A. OSTERMANN. High order splitting methods for analytic semigroups exist. BIT Numer. Math. 49/3 (2009) 527-542.

16. D. HenRY. Geometric Theory of Semilinear Parabolic Equations. Lecture Notes in Mathematics 840, Springer, Berlin, 1981.

17. E. Hille AND R.S. Phillips. Functional Analysis and Semi-Groups. American Mathematical Society, Providence, 1957.

18. T. JAHNKE AND CH. LUBICH. Error bounds for exponential operator splittings. BIT Numer. Math. 40/4 (2000), 735-744.

19. СH. LUBICH. On splitting methods for Schrödinger-Poisson and cubic nonlinear Schrödinger equations. Math. Comp. 77 (2008), 2141-2153.

20. A. LunARdi. Analytic Semigroups and Optimal Regularity in Parabolic Problems. Birkhäuser, Basel, 1995.

21. R.I. McLachlan AND R. Quispel. Splitting methods. Acta Numerica 11 (2002), 341-434.

22. CH. Neuhauser AND M. THALHAMMER. On the convergence of splitting methods for linear evolutionary Schrödinger equations involving an unbounded potential. BIT Numer. Math. 49/1 (2009), 199-215.

23. A. PAZY. Semigroups of Linear Operators and Applications to Partial Differential Equations. Springer, New York, 1983.

24. V.M. PÉREZ-GARCÍA AND X. LIU. Numerical Methods for the Simulation of trapped Nonlinear Schrödinger Systems. Appl. Math. Comp. 144 (2003), 215-235.

25. G. STRANG. On the construction and comparison of difference schemes. SIAM J. Numer. Anal. 5 (1968), 506-517.

26. M. THALHAMmer. High-order exponential operator splitting methods for time-dependent Schrödinger equations. SIAM J. Numer. Anal. 46/4 (2008), 2022-2038.

27. H.F. TrotTER. On the product of semi-groups of operators. Proc. Amer. Math. Soc. 10 (1959), 545-551. 